\title{
Serological response to SARS-CoV-2 vaccination in multiple sclerosis patients treated with fingolimod or ocrelizumab: an initial real-life experience
}

\author{
Guerrieri S. ${ }^{1}$ Lazzarin S. ${ }^{1} \cdot$ Zanetta C. $^{1,2} \cdot$ Nozzolillo A. ${ }^{1}$ Filippi M. ${ }^{1,2,3,4,5} \cdot$ Moiola L. ${ }^{1}$ (1)
}

Received: 31 May 2021 / Revised: 10 June 2021 / Accepted: 11 June 2021 / Published online: 26 June 2021

(c) The Author(s) 2021

\begin{abstract}
Background Recent observations suggest a lack of humoral response after SARS-CoV-2 vaccination in multiple sclerosis (MS) patients treated with fingolimod or ocrelizumab

Objectives To assess serological response to SARS-CoV-2 vaccination in MS patients receiving these disease-modifying treatments (DMTs) in a real-life setting.

Methods Retrospective clinical data collection from MS patients followed at San Raffaele Hospital MS Centre (Milan, Italy). All patients treated with fingolimod or ocrelizumab who had received a complete anti-COVID-19 vaccination course, with no clinical history suggestive of previous SARS-CoV-2 infection and with an available post-vaccination serological assay obtained at least 14 days after vaccination completion were considered for the study.

Results We collected data from 32 MS patients, 16 treated with fingolimod and 16 receiving ocrelizumab. Among the fingolimod group 10 patients $(62.5 \%)$ had a positive serological response after vaccination and among ocrelizumab-treated patients a positive serological test was found in six cases (37.5\%). No relation between serological response and clinical features (i.e., treatment duration, time between vaccination and last treatment dose, and white blood cells count) was identified. Conclusions Our initial real-life experience suggests a variable antibody production in MS patients receiving these DMTs. At present, there are no sufficient data to do not recommend anti-SARS-CoV-2 vaccine in these patients.
\end{abstract}

Keywords Multiple sclerosis $\cdot$ COVID-19 $\cdot$ SARS-CoV-2 vaccination $\cdot$ Fingolimod $\cdot$ Ocrelizumab

SARS-CoV-2 infection has been responsible of an unprecedented pandemic all over the world, with consequences on several social aspects. Several concerns have been raised in relation to an increased susceptibility to COVID-19 in patients affected by Multiple sclerosis (MS), considering the many potential interactions with the immune system,

Moiola L.

Moiola.lucia@hsr.it

1 Neurology Unit, IRCCS San Raffaele Scientific Institute, Milan, Italy

2 Neurorehabilitation Unit, IRCCS San Raffaele Scientific Institute, Milan, Italy

3 Neurophysiology Unit, IRCCS San Raffaele Scientific Institute, Milan, Italy

4 Neuroimaging Research Unit, Division of Neuroscience, IRCCS San Raffaele Scientific Institute, Milan, Italy

5 Vita-Salute San Raffaele University, Milan, Italy disease-modifying treatments (DMTs) and neurological complications described in association with SARS-CoV-2 infection [1]. Recent reviews suggest MS itself does not seem to be associated with an increased severity of such an infection [2,3], with a possible alert for anti-CD20 therapies [4]. Nevertheless, as in the general population, it remains fundamental to predispose adequate preventive strategies, including the recent availability of COVID-19 vaccines.

Inactivated vaccines are generally safe in MS, with the majority of data deriving from previous experiences with flu vaccines [5]. Despite specific information is not yet fully available from clinical trials, no specific safety concerns have emerged for both mRNA and viral vector SARSCoV-2 vaccines in MS patients [6, 7]. COVID-19 vaccination is therefore highly recommended by national societies' guidelines and institutions all over the world. However, it remains to be established whether COVID-19 vaccines are effective in MS, in particular in relation to possible interactions with ongoing DMTs. In this perspective first-line 


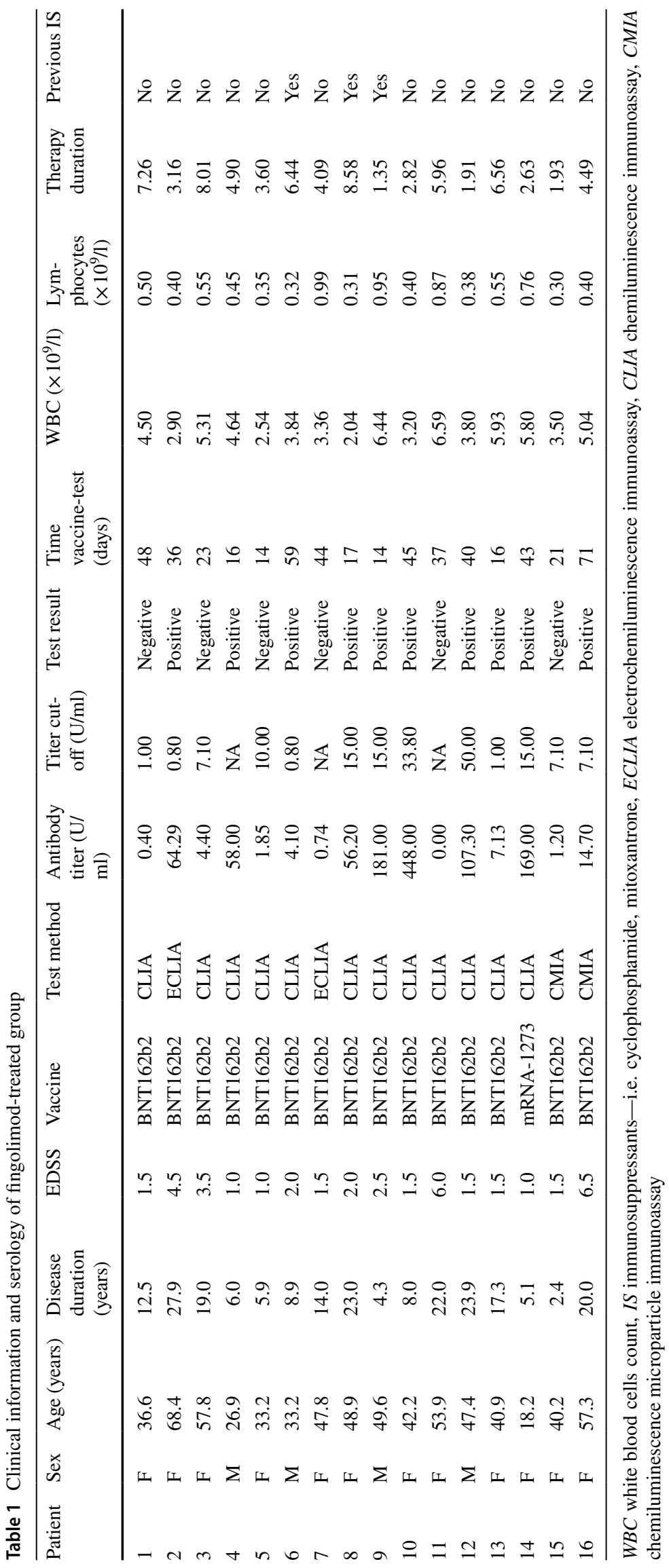




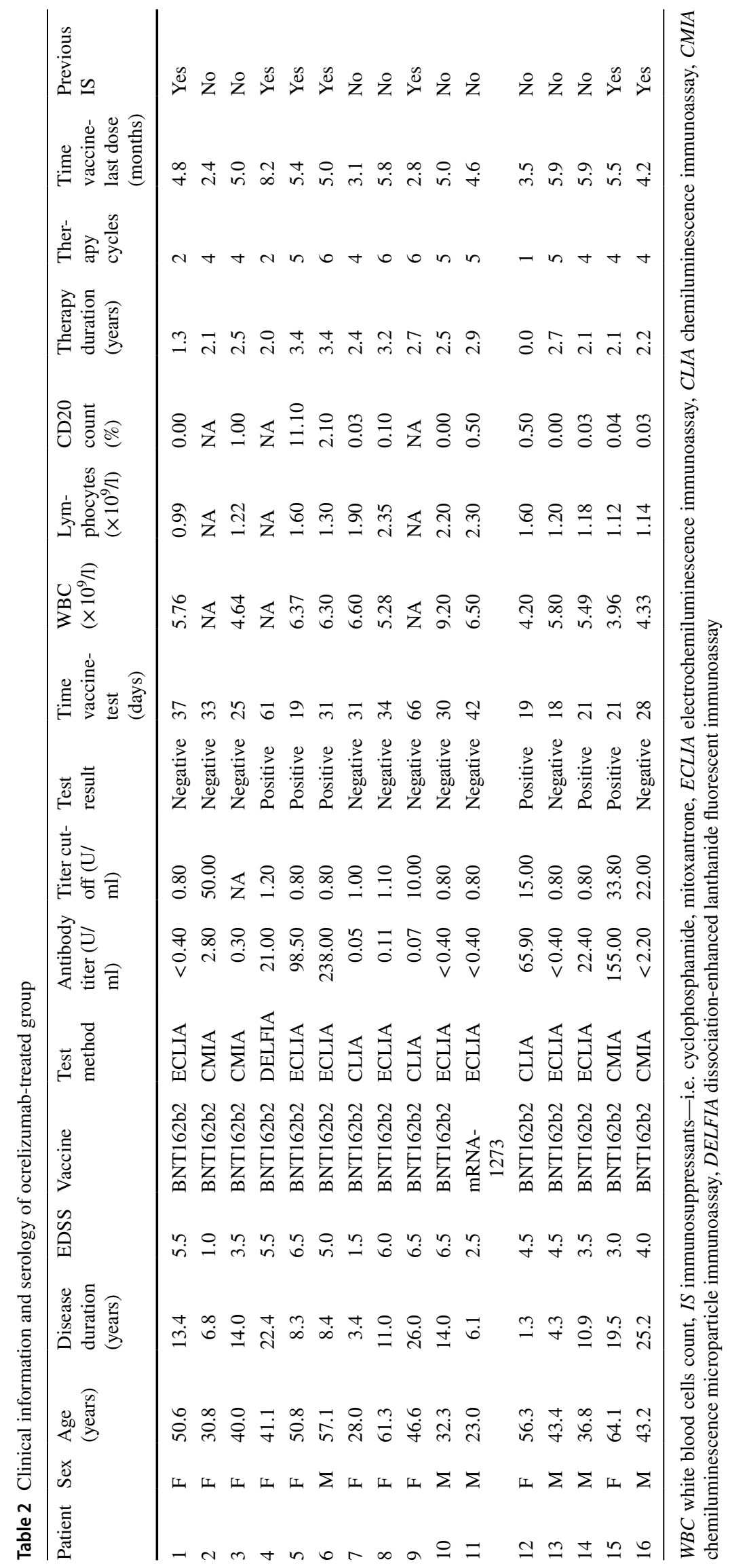


therapies (interferon-beta, glatiramer acetate, teriflunomide and dimethylfumarate) and natalizumab are not expected to compromise vaccine efficacy, while cell-depleting agents (ocrelizumab, rituximab, alemtuzumab and, at least in part, cladribine) as well as modulators of sphingosine-1-phosphate receptor, such as fingolimod, are believed to impact on immune responses after vaccination.

Post-marketing experiences in the field are gradually becoming available. Achiron and colleagues recently assessed humoral response to mRNA COVID-19 vaccines on an Israeli cohort of 93 MS patients-receiving second-line DMTs (23 cladribine, 26 fingolimod and 44 ocrelizumab) and 32 untreated MS patients [8]. After vaccination, a particularly low prevalence of positive SARS-CoV-2 IgG antibodies was detected in ocrelizumab (22.7\%) and fingolimod $(3.8 \%)$ groups. It was, therefore, proposed to avoid COVID19 vaccination in fingolimod-treated patients (considering possible switch to other DMTs) and to schedule vaccination at least 9 months after last treatment dose in patients receiving ocrelizumab. This approach, although very conservative, opens to significant implications about disease activity, with a potential significant risk of relapse deriving from treatment discontinuation or delayed administration. A good serological response was instead detected among cladribine-treated patients (100\%), thus confirming preliminary data available for flu vaccine [9].

We report our initial clinical experience, with the aim to investigate COVID-19 vaccines response in MS patients receiving ocrelizumab or fingolimod. We retrospectively collected data from 32 MS patients followed at San Raffaele Hospital MS Centre (Milan, Italy), 16 treated with fingolimod and 16 with ocrelizumab. All patients received a complete anti-SARS-CoV-2 vaccination course, consisting of two separate doses (30 with BNT162b2 mRNA vaccine produced by Pfizer BioNTech, two with mRNA-1273 vaccine produced by Moderna) and no one had a clinical history suggestive of previous SARS-CoV-2 infection. Anti-Spike protein antibody test was obtained at least 2 weeks (mean 33.1 days, range 14-71) after vaccination course completion. Serology tests have been performed in different laboratories as part of routine clinical assessment in order to evaluate humoral responses to vaccination (detailed techniques for each single patient are reported in Tables 1 and 2). Among the fingolimod group, 10 patients (62.5\%) had a positive serological response after vaccination and among ocrelizumab-treated patients a positive serological test was found in 6 cases $(37.5 \%)$. Complete clinical information is available in Tables 1 and 2; no relation between serological response and clinical features (i.e., treatment duration, time between vaccination and last treatment dose, and white blood cells count) was identified.

Our experience, in comparison to previously published data [8], suggests that humoral response to SARS-CoV-2 vaccination might be highly variable, even in patients treated with fingolimod or ocrelizumab. As a consequence, we believe that SARS-CoV-2 vaccination should be recommended also in MS patients treated with such agents. Indeed, available data are still too limited to suggest treatment discontinuation in order to favor a vaccination response, considering the significant risk of clinical relapse and MRI activity associated to second-line treatment withdrawal (at least in relapsing-remitting MS patients). Furthermore, initial reports relative to other medical conditions causing immunodeficiency suggest the possibility of an efficient cellmediated immunity after vaccination even in the absence of a detectable humoral response [10].

Clearly, this study is not without limitations: the sample size is relatively small and data collection is retrospective, with serological exams performed with different techniques. In addition, we do not have pre-vaccinations serological tests available; northern Italy had a very high prevalence of SARS-CoV-2 infection over the last year, therefore we cannot exclude a previous asymptomatic infection possibly influencing the serological response.

In this historical moment, it is of outmost importance that a very large proportion of the population, including people with MS, adheres to mass vaccination campaigns, in order to get through the current pandemic condition. With the rapid progression of SARS-CoV-2 vaccination programs all over the world, more extensive real-life data from different geographic regions are likely to become available in the near future. The assessment, in the context of prospective clinical trials, of humoral and in particular T cell response to SARSCoV-2 vaccination will be also crucial to tailor the clinical management of MS patients.

\section{Declarations}

Conflicts of interest This article was written in the absence of any intellectual, commercial or financial relationships that could represent a potential conflict of interest.

Ethics and patient consent All patients had signed written consent form for clinical data collection.

Open Access This article is licensed under a Creative Commons Attribution 4.0 International License, which permits use, sharing, adaptation, distribution and reproduction in any medium or format, as long as you give appropriate credit to the original author(s) and the source, provide a link to the Creative Commons licence, and indicate if changes were made. The images or other third party material in this article are included in the article's Creative Commons licence, unless indicated otherwise in a credit line to the material. If material is not included in the article's Creative Commons licence and your intended use is not permitted by statutory regulation or exceeds the permitted use, you will need to obtain permission directly from the copyright holder. To view a copy of this licence, visit http://creativecommons.org/licenses/by/4.0/. 


\section{References}

1. Ellul M, Benjamin L, Singh B, Lant S, Michael BD, Easton A et al (2020) Neurological associations of COVID-19. Lancet Neurol 19(9):767-783. https://doi.org/10.1016/S1474-4422(20)30221-0

2. Louapre C, Collongues N, Stankoff B, Giannesini C, Papeix C, Bensa $C$ et al (2020) Clinical characteristics and outcomes in patients with coronavirus disease 2019 and multiple sclerosis. JAMA Neurol 77(9):1079-1088. https://doi.org/10.1001/jaman eurol.2020.2581

3. Chaudhry F, Jageka C, Levy PD, Cerghet M, Lisak RP (2021) Review of the COVID-19 risk in multiple sclerosis. J Cell Immunol 3(2):68-77. https://doi.org/10.33696/immunology.3.080

4. Sormani MP, De Rossi N, Schiavetti I, Carmisciano L, Cordioli C, Moiola L et al (2021) Disease-modifying therapies and coronavirus disease 2019 severity in multiple sclerosis. Ann Neurol 89(4):780-789. https://doi.org/10.1002/ana.26028

5. Moiola L, Barcella V, Benatti S, Marco Capobianco M, Capra R, Cinque $\mathrm{P}$ et al (2021) The risk of infection in patients with multiple sclerosis treated with disease-modifying therapies: a Delphi consensus statement. Mult Scler 27(3):331-346. https://doi.org/ $10.1177 / 1352458520952311$
6. Kelly H, Sokola B, Abboud H (2021) Safety and efficacy of COVID-19 vaccines in multiple sclerosis patients. J Neuroimmunol 4(356):577599. https://doi.org/10.1016/j.jneuroim.2021. 577599

7. Achiron A, Dolev M, Menascu S, Zohar DN, Dreyer-Alster S, Miron S et al (2021) COVID-19 vaccination in patients with multiple sclerosis: what we have learnt by February 2021. Mult Scler 27(6):864-870. https://doi.org/10.1177/13524585211003476

8. Achiron A, Mandel M, Dreyer-Alster S, Harari G, Magalashvili D, Sonis $\mathrm{P}$ et al (2021) Humoral immune response to COVID-19 mRNA vaccine in patients with multiple sclerosis treated with high-efficacy disease-modifying therapies. Ther Adv Neurol Disord 14:1-8. https://doi.org/10.1177/17562864211012835

9. Roy S, Boschert U (2021) Analysis of influenza and varicella zoster virus vaccine antibody titers in patients with relapsing multiple sclerosis treated with cladribine tablets. Presented at ACTRIMS Forum 2021 as Poster Communication (P071)

10. Bonelli MM, Mrak D, Perkmann T, Haslacher H, Aletaha D (2021) SARS-CoV-2 vaccination in rituximab-treated patients: evidence for impaired humoral but inducible cellular immune response. Ann Rheum Dis. https://doi.org/10.1136/annrh eumdis-2021-220408 